\title{
Erratum to: Expression and Prognostic Significance of p53 in Glioma Patients: A Meta-analysis
}

\author{
Yueling $\mathrm{Jin}^{1} \cdot$ Weizhong $\mathrm{Xiao}^{2} \cdot$ Tingting Song $^{4} \cdot$ Guangjia Feng $^{3} \cdot$ Zhensheng Dai $^{3}$
}

Published online: 14 October 2016

(C) Springer Science+Business Media New York 2016

\section{Erratum to: Neurochem Res (2016) 41:1723-1731 \\ DOI 10.1007/s11064-016-1888-y}

The original version of this article unfortunately contained a mistake in the article note.

The authors wish to correct the article note as: Yueling Jin, Weizhong Xiao, and Tingting Song contributed equally in the preparation of this article.

The online version of the original article can be found under doi:10.1007/s11064-016-1888-y.

Zhensheng Dai

zhenshengdai@126.com

1 Department of Pathology, Shanghai University of Medicine \& Health Sciences, Meilong Road 21,

Shanghai 200237, China

2 Department of Neurology, Shanghai Pudong Hospital Affiliated to Fudan University, Gongwei Road No. 2008, Huinan Town, Pudong New District, Shanghai 201399, China

3 Department of Hematology, Shanghai Pudong Hospital Affiliated to Fudan University, Gongwei Road No. 2008, Huinan Town, Pudong New District,

Shanghai 201399, China

4 Shanghai Putuo District Changfeng Lane Baiyu Community Health Service Center, Caoyang Road 421, Putuo District, Shanghai 200063, China 